\title{
A rush hour towards sexual reproduction: The chromosome dynamics during meiosis
}

\author{
DING DaQiao \\ Advanced ICT Research Institute, National Institute of Information and Communications Technology, Iwaoka-cho, Iwaoka, Nishi-ku, \\ Kobe 651-2492, Japan
}

Received June 15, 2011; accepted August 30, 2011

\begin{abstract}
All Chinese born in the 1960's know the famous movie script "the function of a horse tail". Indeed, there is another famous "horse tail" in the field of basic biology, the premeiotic nucleus in the fission yeast Schizosaccharomyces pombe. It is not only because of its characteristic shape which looks like a horse tail, but also its dramatic oscillatory movement called "the horsetail movement". In the horsetail nucleus, all of the ends of chromosomes, the telomeres, are tightly clustering under spindle pole body (a centrosome equivalent in yeasts). Actually, this horsetail chromosome arrangement is an extreme form of so called "bouquet arrangement", a polarized chromosome arrangement with all the telomeres clustered in a confined area on the nuclear envelope. Bouquet arrangement is widely conserved meiotic prophase chromosomal phenomenon among eukaryotes. From study of the horsetail nucleus in fission yeast, many progresses have been made in the field of meiosis.
\end{abstract}

meiosis, chromosome pairing, fission yeast

Citation: Ding D Q. A rush hour towards sexual reproduction: The chromosome dynamics during meiosis. Chinese Sci Bull, 2011, 56: 3500-3503, doi: 10.1007/ s11434-011-4800-9

\section{An essential unsolved problem in meiosis study}

Meiosis is an essential process for sexually-reproducing eukaryotic organisms, producing haploid gametes from a diploid cell. In this process, one round of DNA replication is followed by two consecutive nuclear divisions to halve the number of chromosomes. The chromosomes during meiosis experience the busiest time during the entire life cycle. To complete meiosis successfully, homologous chromosomes from each parent must first find and align with each other, which are called the homologous chromosome pairing. Later, homologous recombination generates crossovers between the homologous chromosomes; these physical links ensure the correct segregation of chromosomes during the two rounds of nuclear division: reductional segregation of homologous chromosomes in the first di-

email: ding@ nict.go.jp vision, and equational segregation of sister chromatids in the second division. Although the process of homologous recombination and mechanisms for DNA strand exchanging have been extensively studied at a molecular level with even atomic resolution, how homologous chromosomes can find their homologous partners and make pair successfully is still poorly understood. Here, I focus on $S$. pombe to summarize recent advances in functional analyses of telomere clustering and nuclear movement in relation to homologous chromosome pairing, including the underlying molecular mechanisms.

\section{Fission yeast $S$. pombe is a perfect model organism for studying meiosis}

S. pombe cells are maintained in the haploid state during the vegetative growth stage of the lifecycle. Upon nitrogen starvation, haploid cells of opposite mating types conjugate, forming a diploid nucleus through nuclear fusion, the kary- 
ogamy. The process of meiosis normally begins immediately without any intervening mitotic cycles. The horsetail period starts immediately after karyogamy, and continues for about $2 \mathrm{~h}$ preceding metaphase of the first meiotic division (Figure 1). The horsetail period also involves premeiotic DNA synthesis. Two characteristic cytological features in meiotic prophase of $S$. pombe are telomere clustering and nuclear movement. Telomere clustering starts in response to nitrogen starvation and mating pheromone signaling prior to karyogamy [1,2]. During telomere clustering, all of the telomeres originally dispersed on the periphery of the nucleus (Rabl orientation of chromosome, Figure 2) move to a position beneath the spindle pole body and form a tight cluster, while centromeres anchored beneath the SPB in mitotic interphase detach (Figure 2). The telomere clusters from each haploid gamete fuse during karyogamy. After karyogamy, the diploid nucleus elongates about 3 times in length and initiates oscillation from one end to the other in the cell, forming the horsetail nucleus (Figure 1). The horsetail nucleus continuously moves during the entire period of meiotic prophase. This movement is driven by dynamic elongation and shortage of microtubules emanating from the SPB [3]; many motor proteins are also involved to generate this oscillatory movement [4].

\section{Telomere clustering, horsetail movement and recombination are essential for homologous chromosome pairing}

To understand the biological significance of telomere clustering and the horsetail movement during meiotic prophase, pairing of homologous chromosomes was studied in live cells using LacO/LacI-GFP labeling system [5] (Figure 3). Homologous loci exhibited a dynamic process of association and dissociation during the time course of meiotic prophase. Lack both of the horsetail movement and telomere clustering reduced association frequency between homologous chromosomes. Therefore, oscillation of telomerebundled chromosomes efficiently promotes spatial side-byside alignment of homologous chromosomes (Figure 4).

Recombination can also promote the alignment of homologous chromosome loci. In the absence of recombination, homologous interactions at chromosome arms were transient as the spatial distance between roughly aligned homologous chromosomes could not be decreased further [5]. The underlying mechanism of recombination in promoting homologous chromosome pairing is not clear. It is possible that reciprocal recombination (crossing over) and resulting chiasmata provides a stable physical link and converging homologous chromosomes.

Interestingly, the association between homologous centromeres is normal in the absence of recombination, suggesting a role for centromere heterochromatin in recombination independent homologous pairing. The recombination-independent centromere pairing may contribute to improvement of fidelity in achiasmate chromosome segregation [4].

\section{Proper condensation of chromosome axis is essential for homologous chromosome pairing}

In most organisms, chromosome compaction increases in
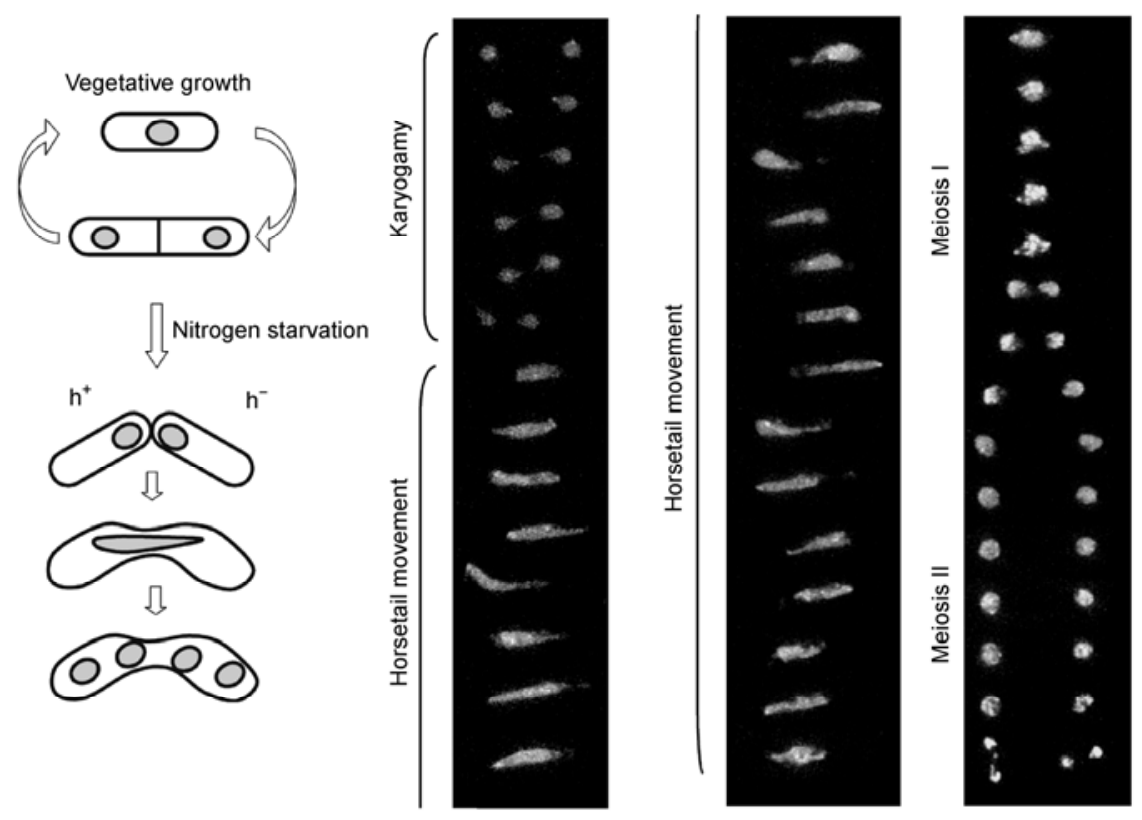

Figure 1 Meiosis in S. pombe. Upon nitrogen starvation, haploid cells of the opposite mating type, $h^{+}$and $h^{-}$, conjugate and enter zygotic meiosis. Time lapse images of nuclei during karyogamy, horsetail movement and chromosome segregations ( 5 min interval) are shown. Chromosomes are visualized with Histone-GFP. 
(a) Rabl orientation
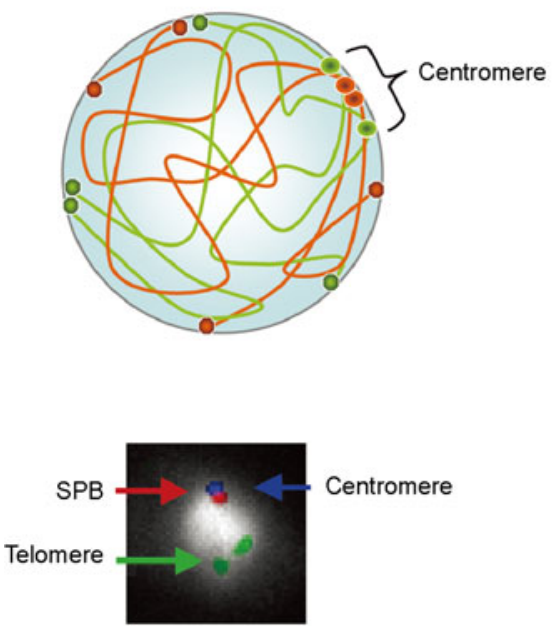

(b) Bouquet arrangement
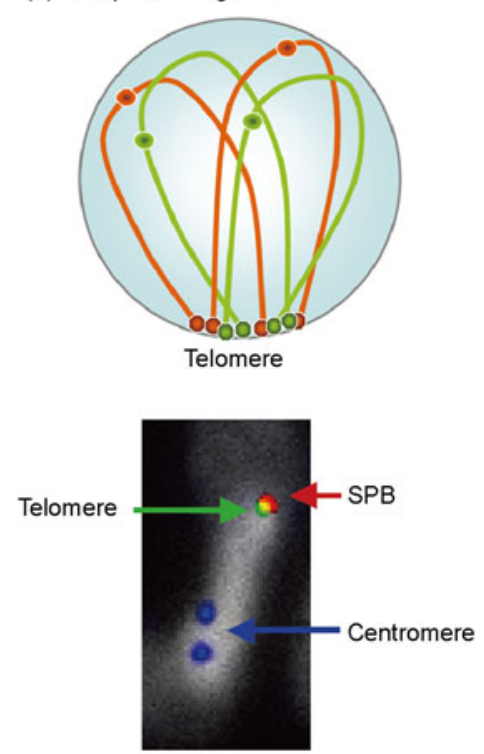

Figure 2 Schematic view and fluorescent images showing the chromosome orientation and the positions of telomeres and centromeres in the nucleus. (a) $\mathrm{Rabl}$ orientation in mitotic cells, where centromeres are clustering near SPB and telomeres are attached to the nuclear envelop. (b) Bouquet arrangement during meiotic prophase, where telomeres are clustering near SPB and centromere are detached from SPB.

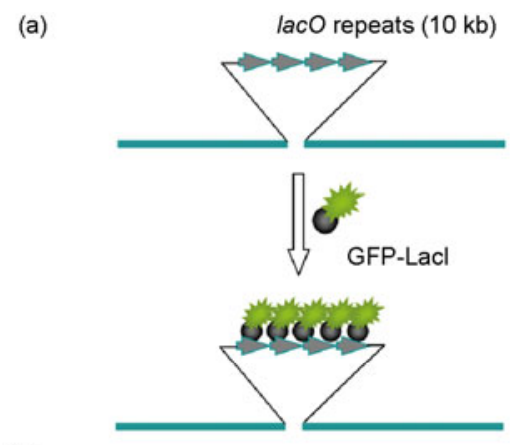

(b)

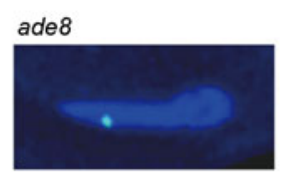

ade6
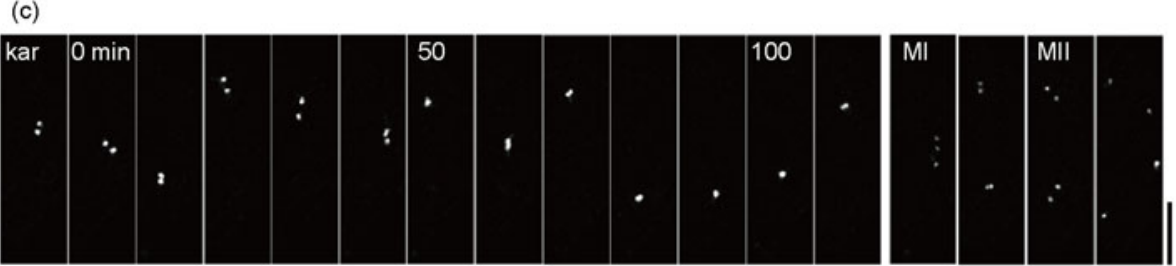

Figure 3 Pairing of homologous chromosome during meiosis. (a) Schematic view showing the lacO/lacI-GFP system. (b) Double stained images of a horsetail nucleus: the blue colour represents DNA stained with Hoechst 33342 and the green dots represent lacO arrays at ade 8 and ade6 gene locus stained with lacI-GFP. (c) Time-lapse observation of the pairing process of ade 8 locus in a living cell. Time in the horse-tail stage is shown. Bar, $5 \mu \mathrm{m}$.

conjunction with the formation of the synaptonemal complex, a tripartite proteinaceous structure. Meiotic cohesins are as major components of the chromosome axis, and play critical roles in the assembly of SC. In $S$. pombe meiosis, the Rec8-containing meiotic specific replaces mitotic cohesion complex, and this replacement is important for the meiosis-specific regulation of sister chromatid cohesion, recruitment of recombination proteins, and for meiotic specific kinetochore orientation. In cells lacking Rec8, chromosomes are under-compacted as if lacking a backbone, and only the leading edge of the nucleus oscillates; a major mass of chromosomes does not follow the movement [6]. In contrast, cells lacking Pds5 (cohesin-associated protein) produce a short over-compacted axis of chromosomes [6]. In both of these mutants, spatial alignment of homologous chromosomes is largely inhibited (Ding \& Hiraoka, unpublished results). Meiotic cohesion complex may contribute to homologous pairing through both recruitment of recombination proteins and establishment of meiosis-specific chromosome architecture. 


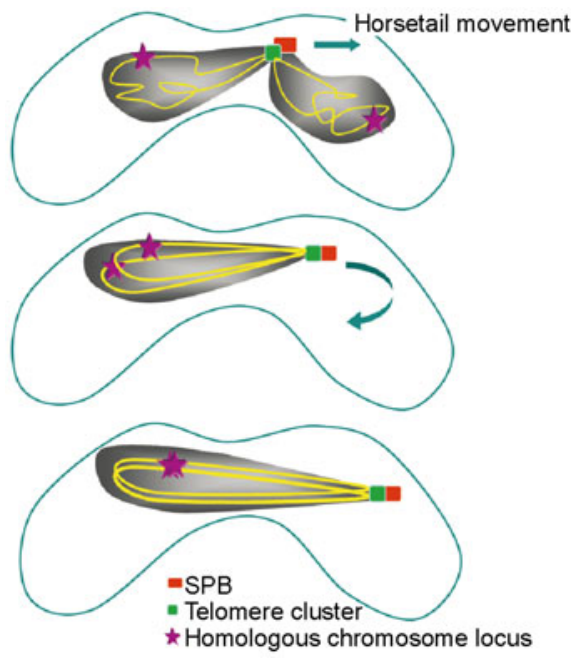

Figure 4 Model of homologous chromosome pairing in $S$. pombe. The telomere-led nuclear movement align homologous chromosome from opposite direction during karyogamy to the same direction in the horsetail nucleus. Spatial distance between homologous loci is dramatically decreased in the horsetail stage.

\section{Hint in homologous chromosome recognition}

Although formation of the bouquet arrangement reduces the spatial distance between homologous chromosomes which could promote the pairing process, it does not directly drive recognition of homologous chromosomes. In S. pombe, we recently uncovered a novel phenomenon relating noncoding RNA to homologous chromosome pairing (Ding, unpublished results), implying that transcribed RNA mediates recognition of the respective DNA region of homologous chromosomes. It is tempting to speculate that particular transcription patterns along each chromosome provide a chromosomal barcode for the recognition of homologous chromosomes. How chromosomes identify their homologous partners remains to be elucidated. The diversity of the underlying mechanisms present in different organisms further increases the complexity of this problem. Studies in $S$. pombe will provide further insight into the mystery of meiosis.

1 Chikashige Y, Ding D Q, Funabiki H, et al. Telomere-led premeiotic chromosome movement in fission yeast. Science, 1994, 264: 270273

2 Chikashige Y, Ding D Q, Imai Y, et al. Meiotic nuclear reorganization: Switching the position of centromeres and telomeres in the fission yeast Schizosaccharomyces pombe. EMBO J, 1997, 16: 193-202

3 Ding D Q, Chikashige Y, Haraguchi T, et al. Oscillatory nuclear movement in fission yeast meiotic prophase is driven by astral microtubules, as revealed by continuous observation of chromosomes and microtubules in living cells. J Cell Sci, 1998, 111: 701-712

4 Ding D Q, Hiraoka Y. Nuclear movement enforcing chromosome alignment in fission yeast-meiosis without homolog synapsis. In: Egel R, Lankenau D H, eds. Genome Dynamics \& Stability (3) Recombination and Meiosis. Berlin: Springer, 2007. 231-247

5 Ding D Q, Yamamoto A, Haraguchi T, et al. Dynamics of homologous chromosome pairing during meiotic prophase in fission yeast. Dev Cell, 2004, 6: 329-341

6 Ding D Q, Sakurai N, Katou Y, et al. Meiotic cohesins modulate chromosome compaction during meiotic prophase in fission yeast. $\mathbf{J}$ Cell Biol, 2006, 174: 499-508

Open Access This article is distributed under the terms of the Creative Commons Attribution License which permits any use, distribution, and reproduction in any medium, provided the original author(s) and source are credited. 